\title{
An Energy-Based Stochastic Model for Wireless Sensor Networks
}

\author{
Yuhong Zhang ${ }^{1}$, Wei $\mathbf{L i}^{2}$ \\ ${ }^{1}$ Department of Engineering Technology, Texas Southern University, Houston, USA \\ ${ }^{2}$ Department of Computer Science, Texas Southern University, Houston, USA \\ E-mail: zhangya@tsu.edu,liw@tsu.edu \\ Received August 2, 2011; revised August 25, 2011; accepted September 6, 2011
}

\begin{abstract}
We propose an energy-based stochastic model of wireless sensor networks (WSNs) where each sensor node is randomly and alternatively in an active and a sleep mode. We first investigate the sensor model and derive the formula of the steady-state probability when there are a number of data packets in different sensor modes. We then determine important sensor's performance measures in terms of energy consumptions, average data delay and throughput. The novelty of this paper is in its development of a stochastic model in WSN with active/sleep feature and the explicit results obtained for above mentioned energy consumption and performance characteristics. These results are expected to be useful as the fundamental results in the theoretical analysis and design of various hybrid WSNs with power mode consideration.
\end{abstract}

\section{Keywords: Energy Efficiency, Wireless Sensor Networks, Energy-Based Stochastic Model}

\section{Introduction}

Recently wireless sensor networks (WSNs) have received more and more attention due to their potential in civil and military applications as well as the advances in micro-electromechanical systems (MEMS) technology [1]. WSNs are composed of a large number of sensors equipped with limited power and radio communication capabilities. They can be deployed in extremely hostile environments, such as battlefield target areas, earthquake disaster scenarios, and inaccessible spaces inside a chemical plant or nuclear facility to monitor environmental changes or other required information. There are a number of recent survey publications [2-9] on WSNs outlining several major directions in the area. Also, there are several papers and references therein which closely related to our current investigation. For example, the paper [10] in 2006 introduced a QoS supporting model and an optimal energy allocation in WSNs; the paper [3] in 2009 discussed the main directions to energy conservation in WSNs; the paper [11] in 2011 investigated several characteristics of active/sleep model in WSNs.

Once a WSN system has been designed, additional energy savings can be achieved using dynamic power management (DPM), which shuts down the sensor node if no events occur. Every component in a node can be in different states; for example, the status of each sensor can be in active, idle, or sleep mode, so the sensor may transmit packets to and receive messages from others. Each node's sleep status corresponds to a particular combination of the component power modes. Mathematically speaking, each sensor will have a finite number of different statuses and the state space of each status is also different. The sensor node stays in each status or state for a random time and then transmits into another status or state and stays for another random duration. A special case is that each sensor will have only two different status, say active and sleep, similar as those in [12-14]. The sensor node alternatively stays in active or sleep status for a probability distributed duration. In this paper, we will concentrate on determining the steady-state probability of data packets in a referenced sensor node and then the sensor's energy consumption and the sensor's performance characteristics.

The rest of this paper is organized as follows. Section 2 provides the description of the modeling under investtigation. Section 3 concentrates on the study of the major performance characteristics, and Section 4 analyzes our numerical results. The final Section 5 provides a conclusion for this paper. 


\section{Description of the Model}

We will consider a wireless sensor network (or part of it) where each sensor may have different characteristics in performance. A sensor may be used as a sink, sensor head nodes or regular sensor node. Without loss of generality, we introduce the assumption in a specific node (or head nose) and may temporally omit the node symbol in this section. The detailed assumptions and notations for a node under investigation of this sensor network are as follows.

- Each node will have two major modes: active and sleep. In an active state, the sensor node is fully working - may generate data, process date (receive and relay/transmit) and keep in idle; in a sleep mode, the node cannot interact with the external world.

- Each node have a finite capacity, say size of $C$, to store the data it generated or forwarded from other nodes for relaying purpose.

- The sensor may stay in phase $R$ of the active mode for a random time with exponential distribution with mean $1 / \alpha$. The sensor then may either move to phase $N$ if there is at least one data packet waiting for processing or move to sleep mode when there is no data packets waiting. In the phase $N$ of the active mode, the sensor node may only process (transmit or relay) data packets with random exponential time with mean $1 / \mu$, and may not be able to generate data or receive any data for relay from other sensors. However, 1) in the phase $R$ of the active mode if the total number of data packet is less than a threshold value $K$, the sensor node may

a) Generate packets according to a Poisson process with rate $\lambda$;

b) Process (transmit or relay) data packets with random exponential time with mean $1 / \mu$;

c) Relay packets coming from other sensors in accordance with a Poisson process with rate $\lambda_{E}$.

2 ) in the phase $R$ of the active mode, if the total number of data packet is more than the threshold value $K$, the sensor node may only have the above function a) and b), and will not be able to relay any packets from neighbor sensor nodes.

- The duration of the sensor in a sleep mode is exponentially distributed with mean $1 / \beta$. When a sensor is in a sleep mode, it may disconnect with external world.

Power consumption models of the radio in embedded devices must take both transceiver and start-up power consumption into account along with an accurate model of the amplifier. The latter actually becomes dominant with small packet sizes and long transition times to receive mode because of frequency synthesizer settle down time. In general, the energy consumed per bit in transmission is given $[10,15]$ in terms of the energy per bit needed by the transmitter electronics (including the cost of startup energy), the receiver electronics, the consumption of the transmitting amplifier to send one bit over one unit distance, the specific distance and the path loss factor etc. In this paper, we will consider the energy consumption in terms of the number the packet transmitted and the sensor mode status, and will use the following notations:

$e_{t r}$ : the transmitter power consumption per data packet in phase $\mathrm{R}$ of the active mode;

$e_{t n}$ : the transmitter power consumption per data packet in phase $\mathrm{N}$ of the active mode;

$e_{a s}$ : the power consumption when sensor switches from the active mode to the sleep mode;

$e_{s a}$ : the power consumption when sensor switches from the sleep mode to the active mode.

\section{Performance Characteristics}

\subsection{Distribution of the Number of Data Packets in Sensor Node}

The purpose of this section is to derive the formula for the steady-state probability of the node when there are $i$ $(i \geq 0)$ packets (including the one being processing and the others being waiting) in the sensor node. Denote by

- $P\left(R_{n}\right)$ as the steady-state probability of the node when there are $n(0 \leq n \leq C)$ data packets in referenced sensor node and the node is in phase $R$ of active mode;

- $P\left(N_{n}\right)$ as the steady-state probability of the node when there are $\mathrm{n}(0 \leq n \leq C)$ data packets in referenced sensor node and the node is in phase $N$ of active mode;

- $P(S)$ as the steady-state probability of the node when the node is in the sleep mode.

In order to reach our result, we need to introduce three stochastic processes. One is the mode status of the node at time $t$, say $I(t)$. The space of this process consists of phase $R$ in active mode, phase $N$ in active mode or sleep mode. The second process is the number of packets in phase $R$ of the active mode node at time $t$, say $X_{I}(t)$. The space of this process is from zero to the maximum capacity of sensor, $C$, when $I(t)$ is at phase $R$ of active mode; The third process is the number of packets in phase $N$ of the active mode node at time $t$, say $Y_{I}(t)$. The space of this process is from one to the maximum capacity of sensor when $I(t)$ is at phase $N$ of active mode. Based on the description of the sensor node as proposed in the previous section and by noting a similar but different consideration in our papers $[16,17]$, we will determine 
that the joint process $\left\{X_{I}(t), Y_{I}(t)\right\}$ forms a multiple dimensional Markov process with the transition rate diagram as in the Figure 1. In this Figure, the cycle notation with $R_{i}$ means that the referenced sensor node is in phase $R$ of active mode and there are $i$ data packets in the referenced sensor node; the cycle notation with $N_{i}$ inside means that the referenced sensor node is in phase $N$ of active mode and there are $i$ data packets in the referenced sensor node; the cycle notation with $S$ means that the referenced sensor node is in sleep mode. By noting above transition rate diagram, the corresponding transition rate matrix, say $Q$, of the constructed two dimensional Markov Process $\left\{X_{I}(t), Y_{I}(t)\right\}$ can now be given by:

$$
Q=\left[\begin{array}{ccccccc}
E_{0} & A_{0} & 0 & \cdots & 0 & 0 & 0 \\
B_{1} & E_{1} & A_{1} & \cdots & 0 & 0 & 0 \\
\vdots & \vdots & \vdots & \vdots & \vdots & \vdots & \vdots \\
0 & 0 & 0 & \cdots & B_{C-1} & E_{C-1} & A_{C-1} \\
0 & 0 & 0 & \cdots & 0 & B_{C} & E_{C}
\end{array}\right],
$$

where the matrices $A_{i}, B_{i}$ and $E_{i}$ are given as follows.

- Matrix $A_{i}(i=1,2, \cdots, C-1)$ refers to an arrival of a data packet, through both sensor's sensing and/or relaying from the neighbor nodes (for $i=0,1, \cdots, K-1$ ) or only through sensor's sensing

(for $i=K, K+1, \cdots, C-1$ ), when there are already $i$ data packets in the sensor, and

a) If $i=0,1, \cdots, K-1, A_{i}$ is a $2 \times 2$ matrix given by

$$
A_{i}=\left[\begin{array}{cc}
\lambda+\lambda_{E} & 0 \\
0 & 0
\end{array}\right] \text {. }
$$

b) If $i=K, K+1, \cdots, C-1, A_{i}$ is a $2 \times 2$ matrix given by

$$
A_{i}=\left[\begin{array}{ll}
\lambda & 0 \\
0 & 0
\end{array}\right] .
$$

- Matrix $B_{i}(i=1,2, \cdots, C)$ refers to a completion of data packet transmission when there are $i$ data packets in the sensor node, and

$$
B_{i}=\left[\begin{array}{ll}
\mu & 0 \\
0 & \mu
\end{array}\right] \text {. }
$$

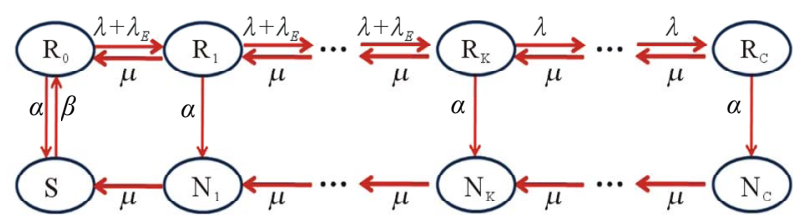

Figure 1. Transition rate diagram of the Markov Process for sensor status.
- Matrix $E_{i}((i=0,1,2, \cdots, C)$ refers to no change in the total number of data packets in the sensor when there are $i$ data packets in the sensor, and

a) if $i=0$, then

$$
E_{0}=\left[\begin{array}{cc}
-\left(\lambda+\lambda_{E}+\alpha\right) & 0 \\
\beta & -\beta
\end{array}\right] .
$$

b) if $i=1,2, \cdots, K-1$, then

$$
E_{i}=\left[\begin{array}{cc}
-\left(\lambda+\lambda_{E}+\mu+\alpha\right) & \alpha \\
0 & -\mu
\end{array}\right] .
$$

c) if $i=K, K+1, \cdots, C-1$, then

$$
E_{i}=\left[\begin{array}{cc}
-(\lambda+\mu+\alpha) & \alpha \\
0 & -\mu
\end{array}\right] .
$$

d) if $i=C$, then

$$
E_{C}=\left[\begin{array}{cc}
-(\mu+\alpha) & \alpha \\
0 & -\mu
\end{array}\right] .
$$

Let $\pi_{0}=\left[P\left(R_{0}\right), P(S)\right]$ and $\pi_{n}=\left[P\left(R_{n}\right), P\left(N_{n}\right)\right]$. For $n=1,2, \cdots, C$, by using Lemma 3 in [18], if we denote $\prod_{i=1}^{n} b_{i}=b_{1} b_{2} \cdots b_{n}$ for matrices $b_{1}, b_{2}, \cdots, b_{n}$, the steady-state probability can be determined by

$$
\pi_{n}=\pi_{0} \prod_{i=1}^{n}\left[A_{i-1}\left(-D_{i}\right)^{-1}\right]
$$

where $D_{i} \quad(i=0,1, \cdots, C)$ are recursively calculated by $D_{C}=E_{C}$ and for $n=0,1, \cdots, C-1$,

$$
D_{n}=E_{n}+A_{n}\left(-D_{n+1}^{-1}\right) B_{n+1},
$$

and $\pi_{0}$ is determined by $\pi_{0} D_{0}=0$ and

$$
\pi_{0}\left[I+\sum_{n=1}^{C} \prod_{i=1}^{n}\left[A_{i-1}\left(-D_{i}\right)^{-1}\right]\right] e=1 .
$$

From above result, if denote by $e_{1}=\left(\begin{array}{l}1 \\ 0\end{array}\right), e_{2}=\left(\begin{array}{l}0 \\ 1\end{array}\right)$ and $e=\left(\begin{array}{l}1 \\ 1\end{array}\right)$, we will have

$$
\begin{gathered}
P\left(R_{n}\right)=\pi_{n} e_{1} \text { for } n=0,1, \cdots, C ; \\
P(S)=\pi_{0} e_{2} ; \\
P\left(N_{n}\right)=\pi_{n} e_{2} \text { for } n=1,2, \cdots, C .
\end{gathered}
$$

\subsection{Energy Consumption Measure of the Sensor}

As long as the result in the formulas (4) and (6) of steady probability is derived, we can find various energy consumption measures in milliwatt-second $\left(\mathrm{mW}^{*} \mathrm{~s}\right)$ of the 
sensor node. We will list the following results to demonstrate how to utilize this formula to obtain the sensor node measures.

- The average energy expended per unit time switching from active mode to sleep mode, denoted by $E_{A S}$. Since the sensor will consume $e_{a s}$ milliwatt ( $\mathrm{mW}$ ) in power each time when the sensor switches from the active mode to the sleep model, and by noting the expected switching number from active model to sleep mode per unit time is $\sum_{i=1}^{C} P\left(R_{i}\right) \alpha$, we will have

$$
E_{A S}=\sum_{i=1}^{C} P\left(R_{i}\right) \alpha e_{a s}=\sum_{i=1}^{C} \pi_{i} e_{1} \alpha e_{a s} .
$$

- The average energy expended per unit time switching from sleep mode to active mode, denoted by $E_{S A}$. Since the sensor will consume $e_{s a} \mathrm{~mW}$ each time when the sensor switches from the sleep mode to the active model, and by noting the expected switching number from sleep model to active mode per unit time is $P(S) \beta$, we will have

$$
E_{S A}=P(S) \beta e_{s a}=\pi_{0} e_{2} \beta e_{s a} .
$$

- The average energy consumption when the sensor node is in the phase $\boldsymbol{R}$ of active mode, denoted by $E_{T R}$. Since the sensor will consume $e_{t r} \mathrm{~mW}$ for transmitting each data packet in phase $R$ of active mode, and the expected number of data packets in the phase $R$ of active mode is $\sum_{i=1}^{C} i \pi_{i} e_{1}$, we will have

$$
E_{T R}=\sum_{i=1}^{C} i P\left(R_{i}\right) e_{t r}=\sum_{i=1}^{C} i \pi_{i} e_{1} e_{t r}
$$

- The average energy consumption when the sensor node is in the phase $N$, denoted by $E_{T N}$. Since the sensor will consume $e_{t n} \mathrm{~mW}$ for transmitting each data packet in the phase $N$, and the expected number of data packets in the phase $N$ is $\sum_{i=1}^{C} i P\left(N_{i}\right)$, we will have

$$
E_{T N}=\sum_{i=1}^{C} i P\left(N_{i}\right) e_{t n}=\sum_{i=1}^{C} i \pi_{i} e_{2} e_{t n}
$$

\subsection{Sensor Performance Metrics}

We now discuss several major sensor performance metrics as follows.

- The average delay of a data packet in the sensor, denoted by $D$. Since the rate of the sensor's sensing data is $\lambda$ and the rate of sensors' relay request from other sensors is $\lambda_{E}$, by using the Little's law [19], we will have

$$
\begin{aligned}
D & =\frac{1}{\lambda+\lambda_{E}} \sum_{i=1}^{K} i\left[P\left(R_{i}\right)+P\left(N_{i}\right)\right] \\
& +\frac{1}{\lambda} \sum_{i=K+1}^{C} i\left[P\left(R_{i}\right)+P\left(N_{i}\right)\right] \\
& =\frac{1}{\lambda+\lambda_{E}} \sum_{i=1}^{K} i \pi_{i} e+\frac{1}{\lambda} \sum_{i=K+1}^{C} i \pi_{i} e .
\end{aligned}
$$

- If we denote the throughput of a sensor node as the average number of the data packets transmitted from the sensor per unit time, denoted by $T_{n}$, then

$$
T_{n}=\sum_{i=1}^{\infty}\left[P\left(R_{i}\right)+P\left(N_{i}\right)\right] \mu=\sum_{i=1}^{\infty} \pi_{i} e \mu=\left[1-\pi_{0} e\right] \mu .
$$

Remark: Based on our above explicit results, we would like to point out that

- As all above measures are determined by several 2dimensional vector or 2-dimensional matrix, one will easily conduct the related numeral analysis.

- As the sensor node has to relay all data packets in the node when the sensor's mode is changed from phase $R$ to phase $N$, the probability that the sensor is in sleep mode should be less than $\beta /(\alpha+\beta)$, and the probability that the sensor is in active mode should be bigger than $\alpha /(\alpha+\beta)$.

\subsection{Wireless Sensor Network Model}

Now, we will briefly introduce our method for analyzing the hybrid WSNs. The whole wireless sensor network consists of all above mentioned sensor node with active mode in $R$ phase and in $N$ phase, and sleep mode. But the sensors' characterization such as energy installed and the period of active and sleep mode in a sensor may different. We will not approximate this sensor network model as an open queueing network as described in paper [20] or [21]. The key idea in our research is to introduce a trigger strategy as we presented recently in papers [22-24]. Specifically, we will consider a protocol mechanism under which a potential relayed message $n$ to a sensor node with phase $N$ in active mode or sleep mode is either lost from the network or is deemed as a relayed message to a neighbor sensor node with certain probability. Most of the routing protocols in mobile ad hoc networks follow this idea. Under our proposed protocol in hybrid WSNs, we may prove that the stationary distribution of the wireless sensor network has a product form. This allows us to derive explicit expressions for relay message rate from a sensor node (in $N$ phase and sleep model) to another node, and the message lost probability for a generated message and relayed message. 


\section{Numerical Analysis}

To verify the validity of the model and our analytical expressions obtained in the previous section, we have done some further numerical analysis in this section. The numerical results for a set of specific parameters for this network are presented in this section. The performance measures considered here are several energy consumptions, package time delay, and the throughput. We assume the data storage capacity of the sensor network is $20(C=20)$ and the threshold value $K$ is 5,10 and 15 respectively. We will observe and compare the effects of various performance measures in the three cases $(K=5$, 10 and 15) versa the rate $\lambda$ of the active sensor node on generating message, and allow the $\lambda$ changes from 0.05 to 0.5 . The other parameters for this sensor network are listed in Table 1.

Figure 2 shows the energy consumption when the sensor node switches from the active mode to the sleep mode. It is clear that the sensor may consume more energy when the generating rate is increase. Also, from this figure, we know that the more the threshold of reserved capacity in a sensor node, the more the energy the sensor node may consume, although the energy assumption does not change too much. Thus, from the viewpoint of minimizing the energy consumption for switching from active mode to sleep mode, it is optimal to minimize the number of data packets which may need relay through the sensor node under investigation. By considering the relay is a vital property for wireless sensor network, it is imperative to find an optimal threshold value of $K$ to leverage the energy consumption.

Figure 3 shows the energy consumption when the sensor node switches from the sleep mode to the active mode. In this case, the energy consumption is not increasing with the sensor's generating rate but decreases a little bit. This is because that the increased generating rate may increase the average staying time of the sensor in state $R$ and $N$, and reduce the number of the sleep over a unit observation time and then reduce the energy consumption of switching from sleep mode to active mode. In addition, this energy consumption is increasing as the threshold of $K$ is increasing. Thus, similar as in the discussion for Figure 1, we also need to find an optimal threshold value of $K$ to leverage the energy consumption.

The curves in Figure $\mathbf{4}$ and Figure 5 show that with the increase of the generating rate $\lambda$, both the energy assumptions in phase $N$ and $R$ will increase because that

Table 1. The specific value of the parameters.

\begin{tabular}{llll}
\hline$\lambda_{E}=0.2$ & $\mu=0.51$ & $\beta=0.05$ & $\alpha=0.1$ \\
\hline$e_{t r}=31 \mu \mathrm{W}$ & $e_{t n}=11 \mu \mathrm{W}$ & $e_{a s}=0.01 \mu \mathrm{W}$ & $e_{s a}=0.5 \mu \mathrm{W}$ \\
\hline
\end{tabular}

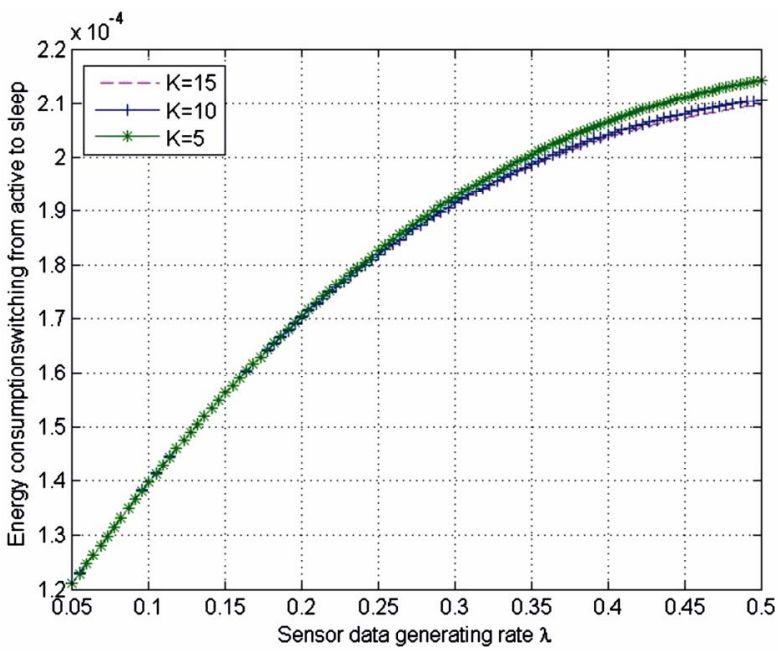

Figure 2. Energy consumption for switching from active to sleep mode.

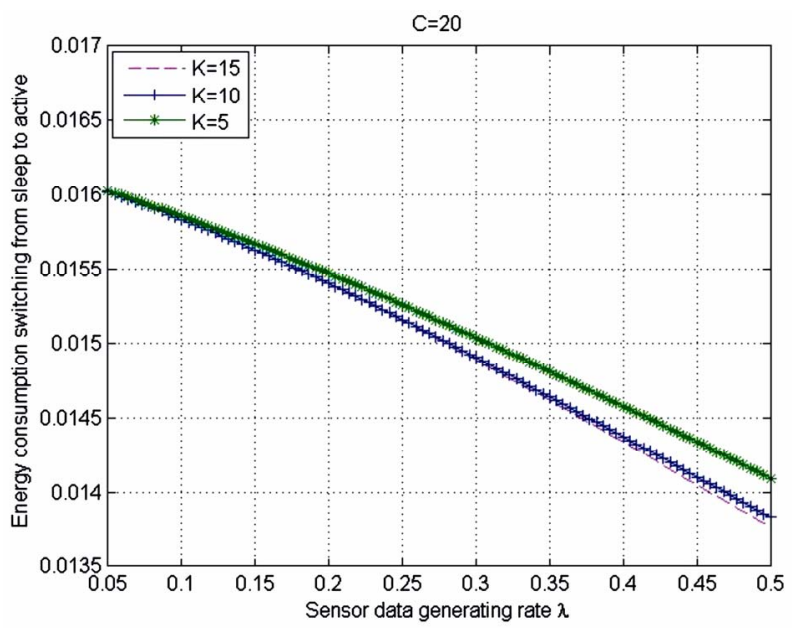

Figure 3. Energy consumption for switching from sleep to active mode.

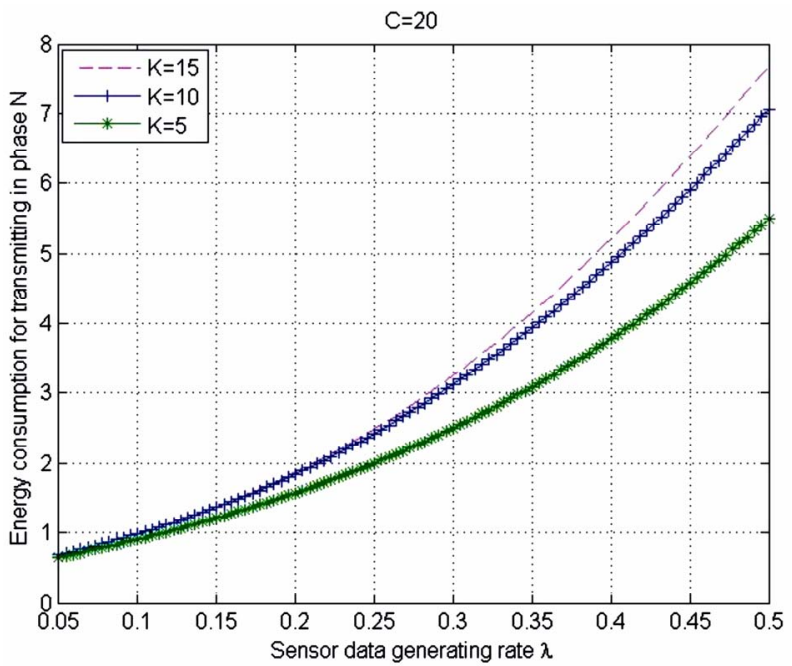

Figure 4. Energy consumption for transmitting in phase $N$. 


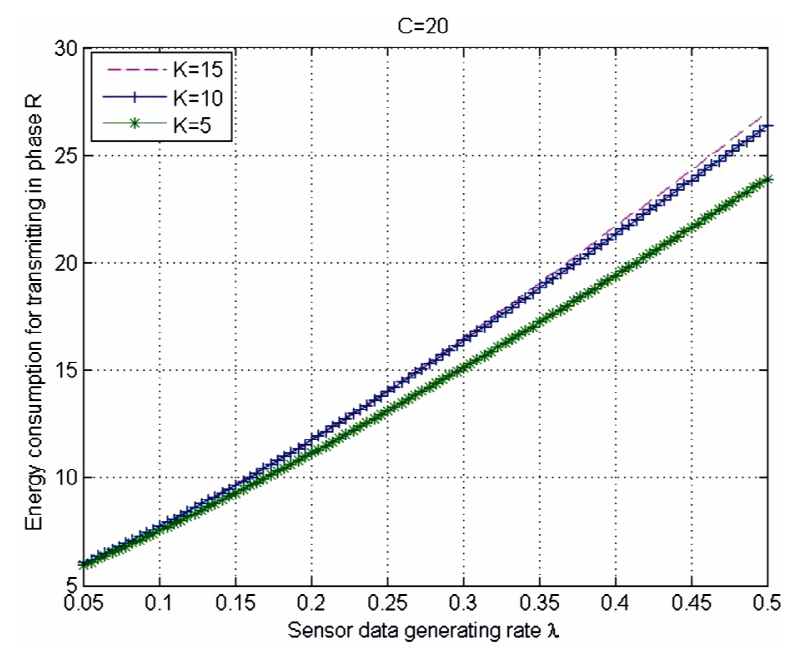

Figure 5. Energy consumption for transmitting in phase $\boldsymbol{R}$.

transmitting more package will spend more energy. Also, these energy consumptions are increased as the sensor generates more data.

The average data delay is depicted in Figure 6 when the sensor generates more data. This delay is obviously increased when either the sensor's generate rate is increased or more relayed message would be processed.

From the Figure 7 on the throughput of the sensor, we know that the throughput will increase when either the sensor's generating rate increases or more relayed message is processed. Based on above analysis, it is clear that a threshold value of $K$ should be identified to leverage the energy consumption, which is in the list of our research agenda.

\section{Conclusions}

In this paper, we investigated several characteristics of

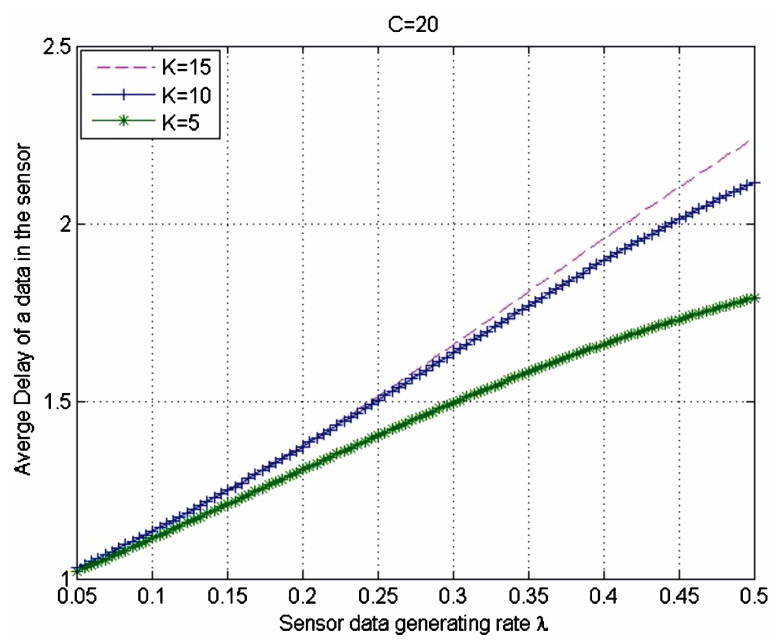

Figure 6. Average data delay vs the increase of sensor node's generating rate.

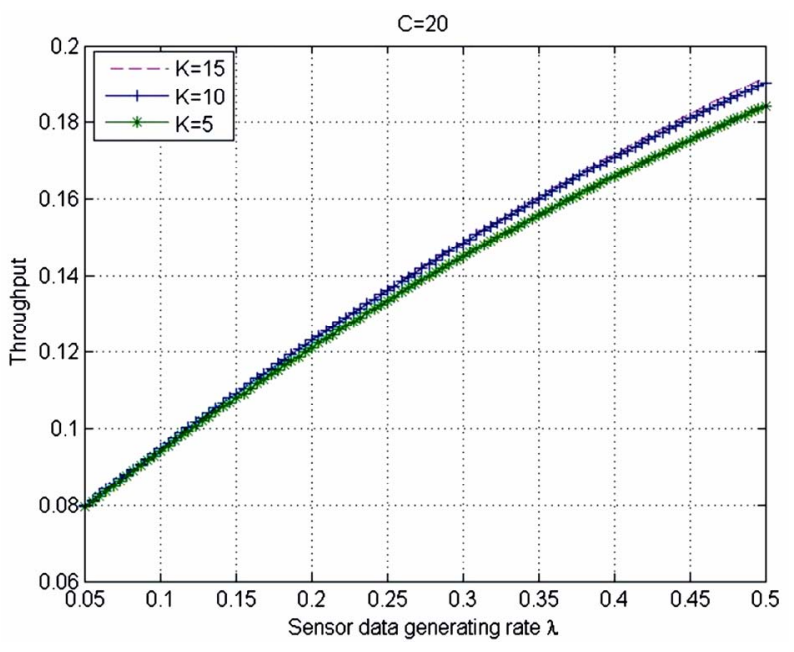

Figure 7. Throughput vs the increase of sensor node's generating rate.

active/sleep mode in wireless sensor network (WSN). We first derived the steady-state probability distribution when the sensor is in different modes and then provided sensor's energy consumption measures and performance characteristics. These technical results may have a great intention to the theoretical analysis of various WSNs with consideration of active and sleep features. We note that the analytic result in the research of WSN is important but often hard to derive. With the analytical method from this paper, we foresee other related results in the future.

\section{Acknowledgements}

This work was supported in part by NSF under grants CNS-1059116 to Yuhong Zhang and HRD-1137732 to Wei Li, and by AFOSR under grant FA-9550-10-1-0128 to Wei Li.

\section{References}

[1] J. W. Gardner, V. K. Varadan and O. O. Awadelkarim, "Microsensors, MEMS, and Smart Devices," John Wiley \& Sons, New York, 2001.

[2] A. A. Abbasi and M. Younis, "A Survey on Clustering Algorithms for Wireless Sensor Networks," Computer Communications, Vol. 30, No. 14-15, 2007, pp. 28262841. doi:10.1016/j.comcom.2007.05.024

[3] I. F. Akyildiz, W. Su, Y. Sankarasubramaniam and E. Cayirci, "Wireless Sensor networks: A Survey," Computer Communications, Vol. 38, No. 4, 2002, pp. 393-422.

[4] I. F. Akyildiz, T. Melodia and K. R. Chowdhury, "A Survey on Wireless Multimedia Sensor Networks," Computer Communications, Vol. 51, No. 4, 2007, pp. 921-960.

[5] G. Anastasi, M. Conti, M. D. Francesco and A. Passarella, "Energy Conservation in Wireless Sensor Networks: A 
Survey," Ad Hoc Networks, Vol. 7, No. 3, 2009, pp. 537-568. doi:10.1016/j.adhoc.2008.06.003

[6] P. Baronti, P. Pillai, V. W. C. Chook, S. Chessa, A. Gotta and Y. F. Hu, "Wireless Sensor Networks: A Survey on the State of the Art and the 802.15.4 and ZigBee Standards," Computer Communications, Vol. 30, No. 7, 2007, pp. 1655-1695. doi:10.1016/j.comcom.2006.12.020

[7] D. Chen and P. K. Varshney, "QoS Support in Wireless Sensor Networks: A Survey," Proceedings of the 2004 International Conference on Wireless Networks, Las Vegas, 21-24 June 2004, pp. 227-233.

[8] K. Sohraby, D. Minoli and T. Znati, "Wireless Sensor Networks: Technology, Protocols, and Applications," Wiley Publisher, Hoboken, 2007.

[9] J. Yick, B. Mukherjee and D. Ghosal, "Wireless Sensor Network Survey," Computer Networks, Vol. 52, No. 12, 2008, pp. 2292-2330. doi:10.1016/j.comnet.2008.04.002

[10] S. Tang and W. Li, "QoS Supporting and Optimal Energy Allocation for a Cluster Based Wireless Sensor Network," Computer Communications, Vol. 29, No. 13-14, 2006, pp. 2569-2577. doi:10.1016/j.comcom.2006.02.007

[11] W. Li, "Several Characteristics of Active/Sleep Model in Wireless Sensor Networks," Proceedings of the 3rd International Workshop on Wireless Sensor Networks: Theory and practice, in conjunction with the 4th IFIP International Conference on New Technologies, Mobility and Security, Paris, 7-10 February 2011, pp. 1-5.

[12] C. F. Chiasserini and M. Garetto, "An Analytical Model for Wireless Sensor Networks with Sleeping Nodes," IEEE Transactions on Mobile Computing, Vol. 5, No. 12, 2006, pp. 1706-1718.

[13] C. F. Hsin and M. Liu, "Randomly Duty-Cycled Wireless Sensor Networks: Dynamics of Coverage?" IEEE Transactions on Wireless Communications, Vol. 5, No. 11, 2006, pp. 3182-3192. doi:10.1109/TWC.2006.04861

[14] Y. Zhao and J. Wu, "Stochastic Sleep Scheduling for Large Scale Wireless Sensor Networks," Proceedings of 2005 IEEE International Conference on Communications, Cape Town, 23-27 May 2010, pp. 1-5.

[15] J. Haapola, Z. Shelby, C. A. Pomalaza-Raez and P. Ma- hoen, "Cross Layer Energy Analysis of Multi-hop Wireless Sensor Networks," Proceedings of the European Workshop on Wireless Sensor Networks, Istanbul, 31 January-2 February 2005, pp. 33-44.

[16] W. Li and A. S. Alfa, "A PCS Network with Correlated Arrival Process and Splitted-Rate Channels," IEEE Journal on Selected Areas in Communications, Vol. 17, No. 7, 1999, pp. 1318-1325. doi:10.1109/49.778188

[17] W. Li and Y. Fang, "Performance Evaluation of Wireless Cellular Networks with Mixed Channel Holding Times," IEEE Transactions On Wireless Communications, Vol. 7, No. 6, 2008, pp. 2154-2160. doi:10.1109/TWC.2008.060634

[18] D. P. Gaver, P. A. Jacobs and G. Latouche, "Finite Birth-and death Models in Randomly Changing Environments," Advances in Applied Probability, Vol. 16, No. 4, 1984, 715-731. doi:10.2307/1427338

[19] P. Harrison and N. Patel, "Performance Modeling of Communication Networks and Computer Architectures", Addison-Wesley Longman Publishing Co., Inc. Boston, 1993.

[20] C. F. Chiasserini and M. Garetto, "An Analytical Model for Wireless Sensor Networks with Sleeping Nodes", IEEE Transactions on Mobile Computing, Vol. 5, No.12, 2006, pp. 1706-1718. doi:10.1109/TMC.2006.175

[21] J. Liu and T. Lee, "A Framework for Performance Model- ing of Wireless Sensor Networks," Proceedings of 2005 IEEE International Conference on Communications, Seoul Korea, 16-20 May 2005, pp. 1075-1081.

[22] X. Chao and W. Li, "Performance Analysis of a Wireless Network with Multiple Classes of Calls," IEEE Transactions on Communications, Vol. 53, No. 9, 2005, pp. 1542-1550. doi:10.1109/TCOMM.2005.855011

[23] W. Li and X. Chao, "Call Admission Control for an Adap- tive Heterogeneous Multimedia Mobile Network," IEEE Transactions on Wireless Communications, Vol. 6, No. 2, 2007, pp. 515-525. doi:10.1109/TWC.2006.05192

[24] W. Li and X. Chao, "Modeling and Performance Evaluation of a Cellular Mobile Network," IEEE/ACM Transactions on Networking, Vol. 12, No. 1, 2004, pp. 131-145. 